\title{
Fault reduction in Nonoscale VLSI interconnection by using carbon nanotubes technology
}

\author{
Behzad Lotfy ${ }^{*}$, Houshang Salehi \\ Department of Engineering, Hakim Nezami High Education Institute, Qouchan, Iran \\ Email address: \\ behzadlotfy@gmail.com (B. Lotfy), Houshang_salhiy@yahoo.com (H. Salehi)
}

To cite this article:

Behzad Lotfy, Houshang Salehi. Fault Reduction in Nonoscale VLSI Interconnection by Using Carbon Nanotubes Technology. Science Journal of Circuits, Systems and Signal Processing. Vol. 3, No. 4, 2014, pp. 26-30. doi: 10.11648/j.cssp.20140304.11

\begin{abstract}
As the VLSI technology scales down, significant challenges are facing the fabrication, modeling, and performance of the integrated circuits. One of the major challenges for the continuiation of the Moore's law is "interconnects" at nano-scale. Interconnects become as important as transistors in the current technologies and will dominate the chip performance at the future technologies. Beside their eletrical performance, their mechanical performnace will be important at the nano-scale. Wires should be resilient enough to cope with Back-End-Of-Line (BEOL) processes. Nano-technology has offered us many solutions to current technology problems. One of the major gift of this technology is Carbon Nanotubes (CNT). CNTs are a promising candidate to replace copper interconnects. They not only provide us with ballistic transport for semiconductors, but also have better mechanical performance. In this paper, we study the mechanical reliability of the CNT interconnects and compare it with their copper conterparts.
\end{abstract}

Keywords: Copper Nano-Wire, Carbon Nanotubes, Fault Tolerance, Interconnections, VLSI

\section{Introduction}

The movement toward the nano-scale circuits brings many new challenges in circuit planning. Smaller sizes of these circuits make it increasingly difficult to control the different physical parameters in the process of the production. The displacement of electricity leads to fixed and periodical interruptions after some cycle of the activity, so that these faults cannot be observed in the process of the production test. This problem of electricity displacement will be increased due to the decrease of the width and the increase of the path change of the system for using nanometer methods. The only acceptable method for encountering such problems is to reinforce the reliability in the process of producing relevant circuits. Thus, we can accept some faults in the chip to obtain an acceptable level of efficiency. Besides, problems of the electricity displacement can be overcome by using additional circuit structure and circuits with dynamic and reconfiguration capability. Additionally, soft faults can be controlled by utilizing static additive methods such as adding the software, information, and time.

\section{General Structure of the Paper}

The progress and development of semiconductor industries in recent years has proved to be important as the global economy and other trades [3]. This development has led to the continual development of advanced systems of the tiny circuits made of the transistors, which are limited to VLSI and ULSI with millions and billions of transistors [3].

One of the key features of the MASFETs is their ability of being used in more complicated constructs. The most logical family in this regard is the complementary metal oxide semiconductor. The tinier transistors allow the chip to be more integrated so that the integrity of the surface increases from hundreds of transistors to the million ones as predicted by Moore in 1965. Moreover, the smaller size of the transistors will cause each transistor works more rapidly with less electricity usage and hence. The total number of transistors in the chip decreases [2, 3].

The most accepted candidates to be replaced with CMOS technology are usually some new emerging technologies and include all probable solution on the basis of the new materials, physic of the tools, new circuit plans, etc. These technologies have some dimensions in common. For example, dimensional scaling has been very limited up to now and its 
development has been very slow the dimensions of normal tolls are in the range of an average molecule. In this scale, unstable states get more and are increased on the surface of the tools so that they no longer guarantee the confident performance of the system.

\subsection{Comparison between the Copper Interconnets and the Nano Tube Carbons}

In making VLSI parts, one of the most important parts that create unique problems in the process of running such circuits is the interconnection. Interconnects are indeed the supplier of information for different parts regarding the output of each previous part. If any problem occurs in it, the output of that part and even the overall circuit can be affected (1).

The nanotube carbon connections can be considered as a good replacement because such connections are considerable capabilities in the electric conductivity, heat transfer or convection, and their fault tolerance (4).

The available connections in any circuit can be divided into two groups:

1. Local connections that are being used in short distances and their delay are less than a few hours cycle.

2. Global connection that is being used for long distances and for distributing the data, hour pulse, power supply, and displacements on the chip and their delay is more than a few hour cycles (5).

We know that copper is highly susceptible for the electrical migration of the global connections and its reliability level decreases at the high amperes $\left(10^{\wedge} 6 \mathrm{~A} / \mathrm{CM}^{\wedge} 2\right)$. Additionally, when the size of a circuit is getting smaller, the crosstalk fault increases and then the parasite and disturbing resistance increases due to the free electron and consequently leads to the delay in global connections. This is the reason why we critically need to replace the global connections and removing the copper in them. Recently, carbon nanotubes have been a point of interest so that in coming future the copper will be replaced with these nanotubes in the latest technologies of global connections. Since the chemical structure of these new connections are confident, they show a high resistance against any sort of electrical migration and any sort of physical ruptures. Even the lowest degree of carbon nanotubes can support high-density currents. For example, the current capability in CNTs after 350 hours of being under the current is approximately $10^{\wedge} 10\left(\mathrm{~A} / \mathrm{CM}^{\wedge} 2\right)$ and in the heat of $250^{\circ}$ their heat conductivity is something between 1700 to 3000 W/M.K. All above, their conductivity features are better than the copper (1).

For on chip several connections, single-walled nanotube (SWCNT) and multi-walled nanotube (MWCNT). Indeed a bundle of MWCNT contains several paralleled SWCNT bundles because of their high conductivity.

\subsection{Nano-Tube Carbons as a Replacement of Copper Nano-Wires}

We compare $\mathrm{CU}$ and CNT bundles in the global connections while in recent years, CNT-based connections have been more increasingly applied although there are few researches that have compared $\mathrm{CU}$ and $\mathrm{CNT}$ connections on the surface of the system.

RLC circuit models are explaned for the connection of CNT and CU bundles and they are used to determine the spreading delay.

We compare CU and CNT bundles in the global connections while in recent years; CNT-based connections have been more increasingly applied although there are few researches that have compared $\mathrm{CU}$ and $\mathrm{CNT}$ connections on the surface of the system.

RLC circuit models are explained for the connection of CNT and CU bundles and they are used to determine the spreading delay.

First, we investigate the delay of the global spreading of the copper wires and connections of CNT packages that have been replaced in the range of $22-45 \mathrm{~nm}$ by the technology process of test nodes. The model of paralleled circuit currents (as explained in previous sections) is used to show the delay of the wires for CNT. It is important to note to the optimal state of the repeat and the placement of CU and CNT.

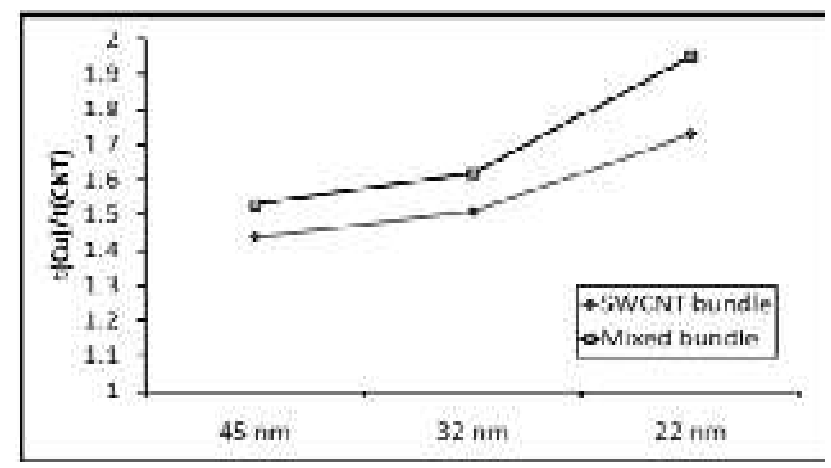

Figure 1. Comparison of the delay of global connections between copper and replaced CNT bundles (6)

Figure 1 shows the ratio of the spreading delay of copper combinations and the spreading delay of CNT bundles. Of course, this issue is true for the overall wires in the length of more than 1 nanometer at the range of 12 to $45 \mathrm{~nm}$ in the nodes technology. It may seem that both SWCNT bundles and the combination of connections of SWCNT and MWCNT bundles have a spreading delay less than the copper (6).

\subsection{Results of the Mechanical Features of the CNT}

In this paper, we have relied on the comparison of the binding energy for studying and comparing the mechanical fault tolerance of the carbon nanotubes and the copper. This is achieved by the relevant simulations, calculations, and comparisons.

\subsubsection{What is Binding Energy}

Binding energy is the force that two atoms have for being bound to each other. The more binding energy, the more solidity of that structure you get. Nevertheless, the higher 
binding energy does not necessarily imply that the relevant structure is always more solid in different states. Thus, the binding energy has to be calculated separately for each phase and for each state of the carbon nanotubes and copper Nanowires so that we can determine which of them is more solid under equal conditions and under equal pressures.

\subsubsection{Binding Energy Analysis}

As mentioned before, the most important part of this paper, is the analysis of the binding energy on the basis of the output of the simulation. To have a better comparison, it will be useful to draw a diagram for the binding energy.

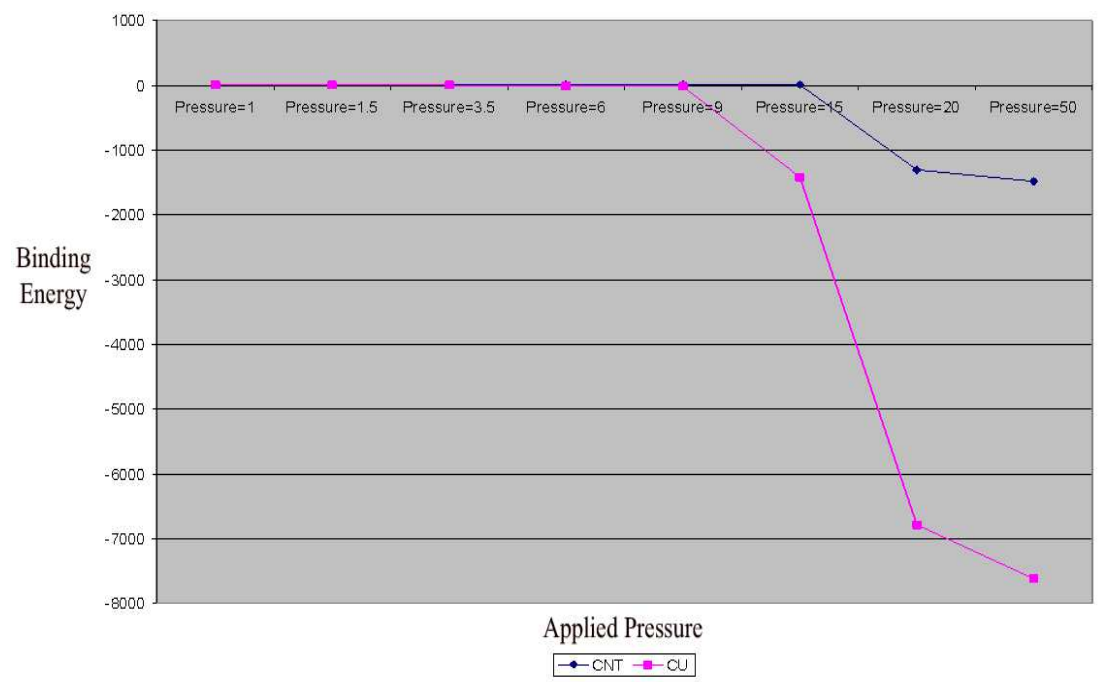

Figure 2. Analysis of binding energy up to 50AT pressure

Referring to the figure 2, we can realize that the level of binding energy for the copper structure is less than the carbon structure and this is true for all applied pressures. Moreover, the connection of carbon structure runs out into a fault area under a higher pressure than the copper structure.
For example, at $15 \mathrm{AT}$ pressure where the carbon nano tubes are still normal, copper nano wires have completely lost their properties. To have a better understanding of the issue and realize the starting point of pressure differences, Figure 3 can be useful.

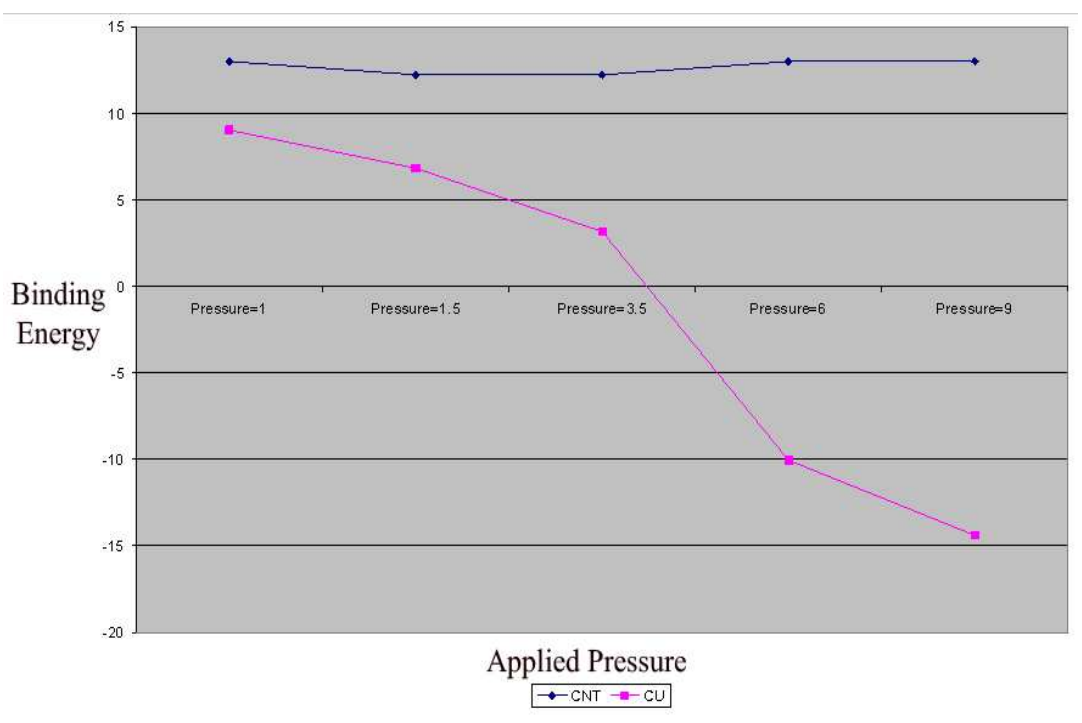

Figure 3. Binding energy up to $9 A T$ pressure

Using this diagram we can observe that the difference level of the binding energy of the two materials begins at 3.5AT pressure. But at the 6AT pressure the copper structure gets completely fault because its level of binding energy becomes negative, while the carbon structure maintains its own nature.

\subsection{The Effect of the Carbon Nano Tube Interconnections}

All simulations and calculations have been done at a separate single level. Now we have to study the effect of these results in a scientifically used set.

When talking about the fault tolerance we have to know the probability of the fault occurance so that we can use these results to improve the effect of the suggested mechanism on the performance of the whole scale of the fault. If the probability of the fault occurance is $10^{-9}$, then in a chip with 
100,000 pieces of these transistors the probability of the lack of the fault is equal to $99.99 \%$. But for a chip with $1,000,000$ transistors, the probability of the lack of the fault is equal to $90.84 \%$ and accordingly, such probability will increasingly reduced by the increase of the parts of each chip (1).

On the other hand, mechanical faults for the intercnnections appear in the form of short circuit faults and partial faults, while the researches show that such fault contains $64 \%$ of all available faults. Now consider the predictions claiming that the whole number of the transitors will be more than 2 billion ones on 2015 . That is, if the currect procedure of the production continues as such, the scale of the correctness of any chip will be significantly lower than the present (6).

Now if we consider the scale of the correctness at the same level of 90.84 , we can conclude that the possibility of fault will be equal to 9.16 where $5.86 \%$ of this possibility is related to the possibility of fault occurance due to the interconnections.

On the yet other hand, we observed that carbon nano tubes are 3 times more tolerant than the copper nano wires only when the chip is damaged under the pressure. Thus the application of this technology will play a significant role in reducing the faults.

\section{Conclusion}

In this paper we studied the effect of using carbon nano tubes as the interconnects in VLSI circuits. Studying and comparing the previous researches and simulations can lead us to the following results:

1. Fault tolerance as one of the most important elements in the manufacturing and producing the chips and circuits have to be considered. Since the application of the chips is now more than before and their application is more sensative than ever, such a consideration is vitally important, because in case of any damage in a circuit, its subsequent loss and repairing and re-running costs of the system will be very heavy.

2. Generally, interconnections have a very important effect on the problem of fault tolerance in VLSI chips and circuits and this effect becomes more and more by the increase of the integrity and development and movement of the circuits toward nano technology.

3. Carbon nano tubes are considered new emerging structures in our today world and the experts and technicians of software and processors development pay special attentions to these structures. The main reason of such attentions is the exclusive and interesting properties of this material. Electrically, carbon nano tubes have a good specification for the connective usages.

4. Carbon nano tubes are very solid and durable in mechanical contexts. This features along with their high capability of integration and the capability of being made in multilayer form have made them useful for promoting many applications.
5. According to the obtained results, we can observe that all three viewpoints about the two structures maintain their early form up to $3.5 \mathrm{AM}$ pressure without any fault and thus they are resistant.

6. At the pressures higher than 3.5AM, the copper gradually gets faulty and at the pressure of 6AT it has completely lost its early state and is gets so-called faulty, while carbon nano tubes still maintains its procedure and does not enters into the fault region.

7. The results of the tests showed that carbon nano tubes lose their early state at the pressures between $15 \mathrm{AT}$ to 20AT and this can be observed in all three cases. In this range of pressure, they lose their role as an interconnect and cannot do their task properly and in fault- free mode.

8. Comparing the pressures that lead to the fault of the two systems we can conclude that carbon nano tubes as the interconnects have the avility of tolerating the pressure three times more that the tolerance of copper nano wires, thus we can claim that these sorces of the fault are three times more tolerant thant the copper nano wires.

As for the strength point of the present study, it can be noted that this study is important in such a way that it has been done in a new interdisciplinary field and modern approaches has been used. The results of the study are also far-reachind and can be applied to different industries and sciences

In addition, the output of the present study can be regarded as the turning point for the future studies.

As far as the weak points of the study is concerned, regarding the fact that the results of the study is new and comprehensive, it has many parameteres and it is suggested that to solve this problem the furthur studies be done in smaller scale.

\section{Acknowledgment}

Hereby we would like to express our deep appreciation to Dr. Sarvari and Dr. Sharifi for their useful guidance and supervisions during the process of this project.

\section{References}

[1] M. Haykel, K. Moselund, D. bouver , 2005, Fault Tolerant Multi Level Logic Decoder for Nanoscale Crossbar Memory Arrays, Integerted Systems Laboratory Switzerland.

[2] M. Heyken Ben Jama, 2009, Fabrication and Design of Nanoscale Regular Circuits, International Conference on Nano Networks.

[3] Y. Jin, F. Yuan, 2003, Simulation of elastic properties of single-walled carbon nanotubes, North Carolina State University.

[4] H. Heidari, S. Mirza Kochaky, M. Babai , 2008, Simulation of Model For Reliability of Nano Wire in Field Programmable Nanowire Interconnect Electronic, Engineering Department of Elmo Sanat University. 
[5] K. Banerjee, N. Srivastava , 2006, Are Carbon Nanotubes the Future of VLSI Interconnections?, San Francisco.
[6] A. Coker, 2008, Performance Analysis of Fault-tolerant NanoElectronic Memories, Texas A\&M University. 